\title{
Gait analysis
}

Interest in the way that the human walks has been evident from early historical times for various reasons. Intellectual curiosity is always a very strong spur in these matters, but the applied aspect of rehabilitation of the disabled has been much to the fore from the middle ages onwards. It is interesting that the photographic measurement of gait took a large step forward owing to a wager relating to the manner of locomotion of horses, which was solved by Muybridge in the late 19th century by a serial photographic technique. At about the same time some rigorous classical studies were being undertaken with a view to optimising the load carrying techniques of the infantryman in the German army! The quantitative analysis of locomotion has much in common with the study of the biomechanics of sports, whose objectives may be thought of as the improvement of performance of athletes of all grades and the selection of appropriate individuals to train for particular skills.

Currently, gait analysis may be considered to be either in research studies or in applied clinical use, though there are obvious overlaps between the two. One example of this is the determination of the parameters of gait for the 'normal' individual-a research exercise conducted so that clinically the performance of a patient may be compared with that of unaffected normal individuals.

In the application of gait analysis to pathological gait it was soon considered important to be able to measure the degree of disability of the patient's locomotor apparatus, frequently using the amount of asymmetry as a guide to treatment or so that different methods of treatment could be assessed on a rigorous basis. From the late 19th century onwards there have been identifications of specific gait deviations corresponding to various clinical disorders. Indeed some of the gait deviations identified subjectively may be of use diagnostically: weakness of the abductor muscles of the hip, for instance, is apparent from the typical lurching gait which it provokes, though not easily differentiated from that due to hip pain.

It is worthwhile considering the parameters which are measured in gait analysis. Generally this is conducted while the test subjects walk in a straight line at uniform speed on a smooth level surface in a clinic or laboratory. A few units use treadmills, though there are theoretical reservations about whether such gait is normal. Useful research data have however been acquired in the negotiation of stairs, ramps, and cross slopes, in cornering, and on general outdoor locomotion involving different surfaces and terrains. For the whole body, measurements include the time to cover a fixed distancethat is, mean velocity, lateral and vertical movement patterns, and variation of forward velocity: both of these leading to kinetic and potential energy calculations.

When the legs are considered independently a much wider range of parameters may be measured: (a) duration of stance and swing phases for each leg; (b) the positions of the joints in three dimensional space; (c) the three dimensional angles of each leg segment in space and the angles of the segments relative to each other and to the trunk in flexion, abduction, or axial rotation. These measurements allow the calculation of time related factors such as angular velocities or accelerations; and $(d)$ the angles of arm movement corresponding to gait and the corresponding trunk displacements.

The foregoing parameters relate to linear or angular displacements. Where it is possible to measure force in any way then values of the forces and stresses developed in muscle, ligament, bones, and joints may be predicted. Force measurement by transducers incorporated in prostheses or orthoses allows the prediction of pressure developed between the devices and the body tissues with which they are in contact.

Recently, measurements of electromyographic activity have been used to predict values of forces in specific muscles, such as soleus and gastrocnemius, but this technique is not generally applicable in other anatomical regions where more than two muscles are agonists.

The wide range of measurements which can be made implies that 'gait analysis' means many different things to different people. For some it may be undertaken subjectively by looking for 'gait deviations', and a list of descriptors has been developed for the phenomena observed, such as 'load sparing' 
or 'antalgic' (although these two may not correspond), 'vaulting', 'hiking', etc. As a wide range of quantities has been identified for measurement so many different instruments have been developed for this purpose. The measuring tape and stopwatch should not be ignored. Cine photography and video recorders are in themselves useful for visualisation of the movement patterns, and the television camera and its many variants form the basis of automated systems for the registration and measurement of the movement of skin mounted markers in three dimensions. All users are aware of the artefact of skin movement relative to the underlying skeleton, though few specifically analyse it. The problem with most systems of this type is that they are not used to measure axial rotations of body segments. As a consequence, for instance, the turning moments in the sagittal plane at the knee may be viewed as corresponding to flexion/extension, assuming a mediolateral orientation of the axis of the knee. When outward rotation of the limb is present a substantial component of this moment may correspond to adducting loading with quite different effects on the structures of the knee. Thus, as always, the sophistication and quantitative sensitivity of the measurement systems may suggest an intrinsic accuracy which may not be present.

The alternative means of movement measurement are by body fixed devices such as goniometers. To the errors due to skin movements for these devices must be added those corresponding to the requirements for accurate alignment with the anatomical joint axes. The depth of the hip joint below the skin surface, the change of knee axis position with increasing flexion, and the oblique orientation of the anatomical axes at the ankle constitute some of the measurement problems. Some newly developed flexible strip transducers relax the requirement for joint axis orientation, though these devices are still subject to the skin/skeleton movement error. In the classical studies of gait conducted at the University of California (1947) bone pins were inserted to act as markers of the skeletal positions in walking. About $50 \%$ of the test records were discarded because of pain or loosening of the pins. Forty years later it appears unlikely that these heroic investigations will be repeated!

Many other scientific parameters have been proposed for the measurement of body segment movement, such as polarised light or electromagnetic transducers, accelerometers, and ultrasound. Although each of these has particular merits, they have not been widely adopted for gait analysis. In some laboratories grids of wire have been incorporated in the walking surface, so that electrical contact or pressure may allow the registration of the positions of the feet in gait. This is useful for determining the temporal parameters but requires other instrumentation to register the positions ofo markers in the leg.

The measurement of ground to foot force has $\frac{\overline{\bar{N}}}{\overrightarrow{2}}$ been part of sophisticated gait analysis systems for $\mathbb{Q}$ many years. For research use most experimenters use a platform of such a size that it can record ${ }^{\infty}$ information from a single step of one foot, though it. is not easy to ensure that the patient or test subject $\overrightarrow{\vec{\omega}}$ places one foot only completely on the platform while 'walking normally'. Such a platform will generally give six signals corresponding to the components of ground force forwards, upwards, and laterally, together with the moments of the resultant force about the vertical, forward, and lateral axes. $c$ Other types of equipment allow the measurement of o successive left and right foot contacts, though such ${ }_{-}$ systems generally impose the constraint that the left 3 and right feet should not cross over the centre line of $\frac{\vec{L}}{\bar{O}}$ the walking direction. Observation of normal gait $\stackrel{\rho}{\triangle}$ indicates that for some people this may be $\mathrm{a}_{\overrightarrow{0}}$ disturbing constraint.

The discriminating feature of gait analysis systems is frequently the method of data display and anyo necessary preprocessing. Goniometers can be connected to plotters to give immediate output of angle to time or angle to angle diagrams, such as hip

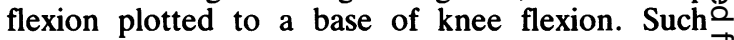
diagrams may be useful for recording the range of $\overrightarrow{\overrightarrow{0}}$ angular movement at these joints, and thus illustrate 3 abnormalities and record asymmetries, giving $\mathrm{a} \rightleftharpoons$ quick record for the observation of progress with treatment. Likewise, in those laboratories having foot contact sensors immediate records can be given:of walking cadence, stance and swing phase times, stride length, and spatial asymmetry.

Multistep force platforms, such as that at Wrightington Hospital, allow a comparison of the vertical components of ground force on the left and right ${ }^{\supset}$ feet, again measuring symmetry of limb loading. With a force platform, video camera, and computing power available it is possible to produce a television ${ }^{N}$ picture of the test subject walking over the forcen platform with the sagittal plane projection of the ground to foot force vector superimposed. Alterna- $\omega$ tively, viewing from the front, the coronal plane projection of the vector can be seen. As previously? mentioned, rotation of the limb segments maye distort the information provided by this system but; generally, it offers considerable insight into the manner of load transmission in the leg. For instanceed if, in the coronal view, the line of the force vector is seen to be near the hip joint for most of the cycle, $\frac{0}{2}$ pain in the hip or weakness in the abductor musclee system may be expected. Alternatively, early move? 
ment of the vector forwards in the sagittal plane can be an indication of non-function of anterior tibial muscles. Thus this sytem may be clinically useful for assisting diagnosis and assessing changes due to treatment. In the case of a patient requiring an ankle-foot orthosis the system may be used to verify that its alignment is improving the ground force vector position relative to the knee joint, reducing the tendency to mediolateral deformity or to hyperextension.

Gait analysis systems using six-quantity force platforms in association with three dimensional spatial measurement systems can analyse the loads transmitted between adjacent body segments at the ankle, knee, and hip joint. As the foot is generally enclosed in footwear and frequently is loaded both on the heel and forefoot these systems can give only restricted information on the mechanics of the joints of the foot. Pedobarograph systems giving the dynamically changing pattern of pressure distribution between ground and the unshod foot may be used to analyse foot loading further. This author is not convinced, however, that gait analysis in bare feet is very relevant to the activities of daily living in the normal adult in the Western World, and although the system records shoe to ground pressures, it cannot indicate the loads transmitted to the foot by the upper.

If the loads transmitted between thigh and trunk are known, estimates can be made of the forces in the relevant muscles, and thus the force transmitted at the hip joint can be predicted both in magnitude and direction. Similar analyses can be undertaken for the knee and ankle joint forces. Because of the tensions developed in the relevant muscles and ligaments these joint forces are generally much higher than body weight during much of the stance phase of walking. Such analyses allow a definition of the loads due to walking which may act on implants such as joint replacements: the information is unlikely, however, to have a direct effect on the treatment of individual patients. It should be apparent also that implants will be subjected to a spectrum of loads not developed in level surface locomotion. Cornering, obstacle avoidance, and negotiation of stairs and ramps develop different values of joint loads, applied in different directions.
To summarise, most researchers know clearly the information which they wish to acquire in their investigations, and the selection of equipment and specification of data to be acquired are usually easily determined. For clinical gait analysis the objective must be clearly specified at the outset. Overoptimistic purchases of sophisticated equipment have frequently led to disappointment, disillusion, and rejection of the concept of gait analysis.

If general walking ability is to be assessed there is much to commend a stopwatch for timing progress round a set path. Range of movement at hip, knee, or ankle joints can be assessed by goniometers. Balance of loading on the two legs may require force platforms but may be inferred from lateral movements of the trunk. Function of specific muscles may be assessed by electromyography. Whatever measurement system is used it will be necessary to set up a data base of normal function, or of the optimal function achieved by patients at identified stages of rehabilitation. Only in this way can quantitative measurements on individuals be assessed. The alternative is to make measurements on patients at each clinical visit so that changes in function due to rehabilitation or progress of disease can be identified.

The advantage of clinical gait analysis is the quantified record which it allows. The disadvantage of many systems currently is the time and manpower required to prepare and conduct the patient through the procedures in a normal gait. Gait in the clinic may be quite different from that when hurrying to catch a bus! As in everything there are no easy solutions, but there is no doubt that wisely chosen equipment, used with care and thought, allows better assessment of the patient.

Bioengineering Unit,

J P PAUL

University of Strathclyde

Further reading

Grieve D W, Miller D, Mitchelson D, Paul J P, Smith A J. Techniques for the analysis of human movement. London: Lepus, 1975.

Paul J P. Gait analysis. In: McAinsh T F, ed. Physics in medicine and biology encyclopaedia. Oxford: Pergamon, 1987: 352-8.

Winter D. Biomechanics of human movement. New York: Wiley, 1979. 